\title{
Communication \\ Unusual Polarization Relation between Single-Mode Lasing Emission and Excitation Laser from an Evanescent-Wave Pumped Micro-Cavity Laser
}

\author{
Yuchen Wang ${ }^{1, *+\dagger}$, Hongsheng $\mathrm{Li}^{1,+}$, Shu $\mathrm{Hu}^{2}$, Heng $\mathrm{Li}^{2}$ and Chuanxiang Sheng ${ }^{2, *}$ \\ 1 School of Science, Qingdao University of Technology, 777 E Jialingjiang Rd, Qingdao 266525, China; \\ lihongsheng1123@qut.edu.cn \\ 2 School of Electronic and Optical Engineering, Nanjing University of Science and Technology, \\ 200 XiaoLingWei, Nanjing 210094, China; 116104000499@njust.edu.cn (S.H.); hengli@njust.edu.cn (H.L.) \\ * Correspondence: wyc1990@qut.edu.cn (Y.W.); cxsheng@njust.edu.cn (C.S.) \\ + These authors contributed equally to this work.
}

Citation: Wang, Y.; Li, H.; Hu, S.; Li, H.; Sheng, C. Unusual Polarization Relation between Single-Mode Lasing Emission and Excitation Laser from an Evanescent-Wave Pumped

Micro-Cavity Laser. Photonics 2021, 8 , 66. https://doi.org/10.3390/ photonics 8030066

Received: 30 January 2021

Accepted: 22 February 2021

Published: 26 February 2021

Publisher's Note: MDPI stays neutral with regard to jurisdictional claims in published maps and institutional affiliations.

Copyright: (c) 2021 by the authors. Licensee MDPI, Basel, Switzerland. This article is an open access article distributed under the terms and conditions of the Creative Commons Attribution (CC BY) license (https:// creativecommons.org/licenses/by/ $4.0 /)$.

\begin{abstract}
Using a fiber of that is $125 \mu \mathrm{m}$ in diameter in rhodamine 6G ethanol solution, controllable multi- and single-whispering-gallery-mode (WGM) optofluidic lasers based on evanescent-wavecoupled gain are both available. With multi-mode WGM emission, lasing emission with almost pure TM (transverse magnetic) or almost TE (transverse electric) modes can be obtained when the pump laser has an electric field parallel (perpendicular) to the fiber axis, i.e., the polarization direction of output laser is the same as that of the pump laser. On the other hand, when the laser emission is single-mode, the TE output laser always emerges firstly above lasing threshold, then keeps TE mode while the pump laser's intensity increases with polarization direction perpendicular to the fiber axis; on the contrary, TE emission will dwindle relatively, while the TM emission emerges and dominates the spectra, when the pump laser's intensity increases with polarization parallel to the fiber axis. Our work proves that controlling the leakage of the evanescent wave from high-Q microcavities is crucial for both modes of lasing emission and its polarization.
\end{abstract}

Keywords: polarization; microcavity lasers; single mode; whispering gallery mode

\section{Introduction}

Recently, whispering-gallery-mode (WGM) optical microcavities have been extensively studied in nonlinear optics [1,2], biosensing [3-5], integrated optics [6], low threshold lasers [7-10] and other research areas, due to their ultrahigh-quality factor $(Q)$ and small volumes. In addition, when microcavities, including Mie resonators, work in the strong coupling regime, mixing light-matter state such as polaritons could dominate optical properties of system, resulting in novel phenomena and applications, including Bose-Einstein condensation of exciton polaritons and polariton laser without inversion [11-14]. On the other hand, normally WGMs were multimode. To achieve single-mode emission in WGM, many efforts, including Vernier Effect and Parity-Time Symmetry breaking, had been used [15-17]. Our group has observed single-mode WGM emission in $125 \mu \mathrm{m}$ diameter micro cylindrical cavity based on evanescent wave coupled gain, which was experimentally proved to be due to the leakage from the fiber to the cuvette wall [18]. The symmetry breaking can introduce unique properties in optical physics $[19,20]$. Moreover, the polarization of the emission is an important property of WGM lasers. The effect of pumping polarization on the polarization of WGM emission has been explored in several works [21-24]. It has been demonstrated that the polarization of pumping light determines the polarization of the vibration states of excited molecules and, consequently, the polarization properties of lasing emission [21-24]. However, the relations between the pump's and emission's polarization are usually obtained in multimode WGMs [21-24]. The influence of leakage 
on the polarization dependence between excitation and emission, especially the single mode emission, is unknown.

In this work, the relation between pumping polarization and the polarization of the single mode WGM laser was investigated. It is worth noting firstly that the single mode is different from the multimode one, in which the polarization direction of the output laser is found to be the same as that of the pump laser. In the single-mode situation, no matter whether the polarization of pumping laser is parallel or perpendicular to the fiber axis, the TE mode output laser, for which the polarization is perpendicular to the fiber axis, always emerges first above the lasing threshold. However, at high pump intensities, the polarization direction of the output laser is basically the same as that of the pump laser. This means that TE emission will decrease relatively while TM emission emerges and dominates the spectra when the pump laser's intensity increases with its polarization parallel to the fiber axis.

\section{Materials and Methods}

\subsection{Sample Preparation}

The $2.5 \mathrm{mg} / \mathrm{ml}$ Rh6G solution was made by dissolving $2.5 \mathrm{mg}$ Rh6G in $1 \mathrm{~mL}$ ethanol and was put in a quartz cuvette. A segment of an optical fiber (radius $r=62.5 \mu \mathrm{m}$ ) was used as a cylindrical cavity. The refractive index (RI) of the solution is 1.361, and the RIs of the cuvette and fiber are both $\sim 1.455$. The bare fiber was cleaned carefully, using ethanol. Then it was dipped into the solution, vertically resting against the cuvette front wall, due to the capillary force, with a very thin layer of solution between them.

\subsection{Optical Setup}

The sample was pumped by an Nd:YAG $532 \mathrm{~nm}$ Q-switched laser with $10 \mathrm{~ns}$ pulse duration, operating at repetition rate of $10 \mathrm{~Hz}$. A pair of polarizers was set in front of the laser. The first polarizer was used to adjust the laser intensity, by varying its angle. The second one was used to determine the polarization of the excitation laser. Then the laser beam was focused by a lens with a focus length of $10 \mathrm{~cm}$, to obtain a circle excitation spot on the sample with diameter of $\sim 100 \mu \mathrm{m}$. The emission of the sample was collected by a couple of lenses, using back scattering geometry, and the polarization of the emission was also checked by a polarizer.

As denoted in Figure 1, for the polarization of excited laser beam, it is either perpendicular ( $p$-polarized) or parallel (s-polarized) to the fiber axis, the emission is also separated as being either TE and TM modes, respectively. A spectrometer mounted with a CCD (Charge-coupled Device) was used to record the spectrum, with a resolution of $\sim 0.1 \mathrm{~nm}$.
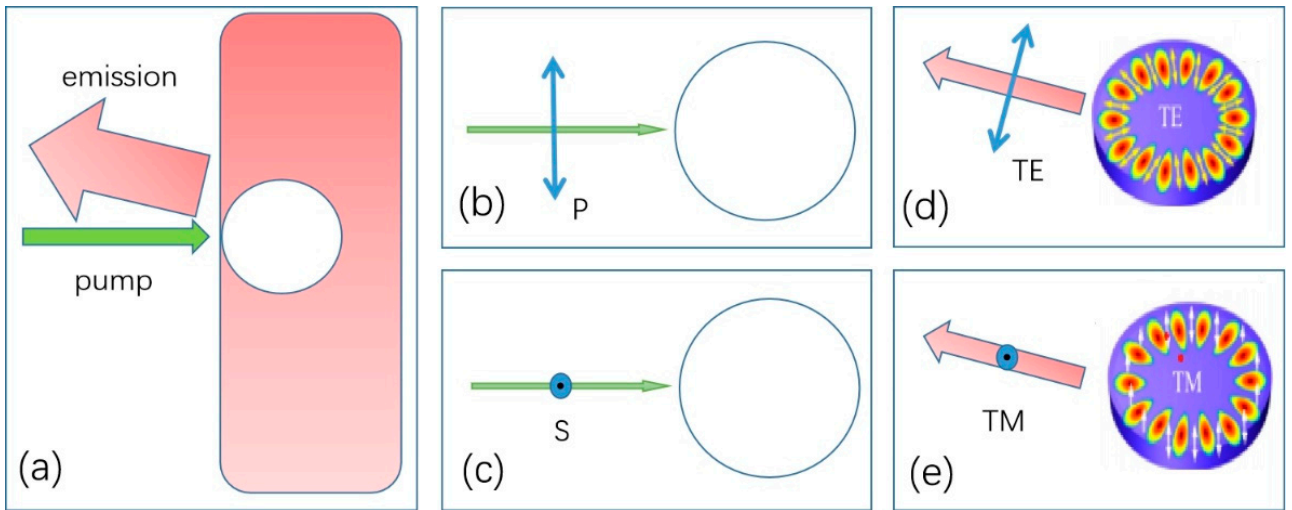

Figure 1. (a) Diagram of experimental setup; white disk represents fiber; (b) and (c) are schematic diagrams of the cavity pumped by laser with $p$-polarized (perpendicular) and s-polarized (parallel) light, respectively; (d) and (e) are diagrams of emission whispering-gallery modes (WGMs) in the cavity with TE and TM modes, respectively. 


\section{Results and Discussion}

Because of the existence of capillary force, the fiber would stick to the cuvette wall. Due to the competition between optical coupling of fiber/cuvette-wall and fiber/surroundingsolution, there would be two situations for laser emission: One is the evenly spaced multimode WGM emission [21-27], and the other is single-mode emission [18]. When the optical coupling between fiber/solution dominates the leakage loss of WGM in the fiber (normally the fiber may not stick to the cuvette wall very well because the fiber surface is not clean or is damaged) [18], the spaced multimode WGM emission would be observed, as shown in Figure 2. When the fiber was pumped by s-polarized light, there were only TM modes existing in the emission spectra, for which the polarizations of both pump and output lasers are both parallel to the fiber axis.
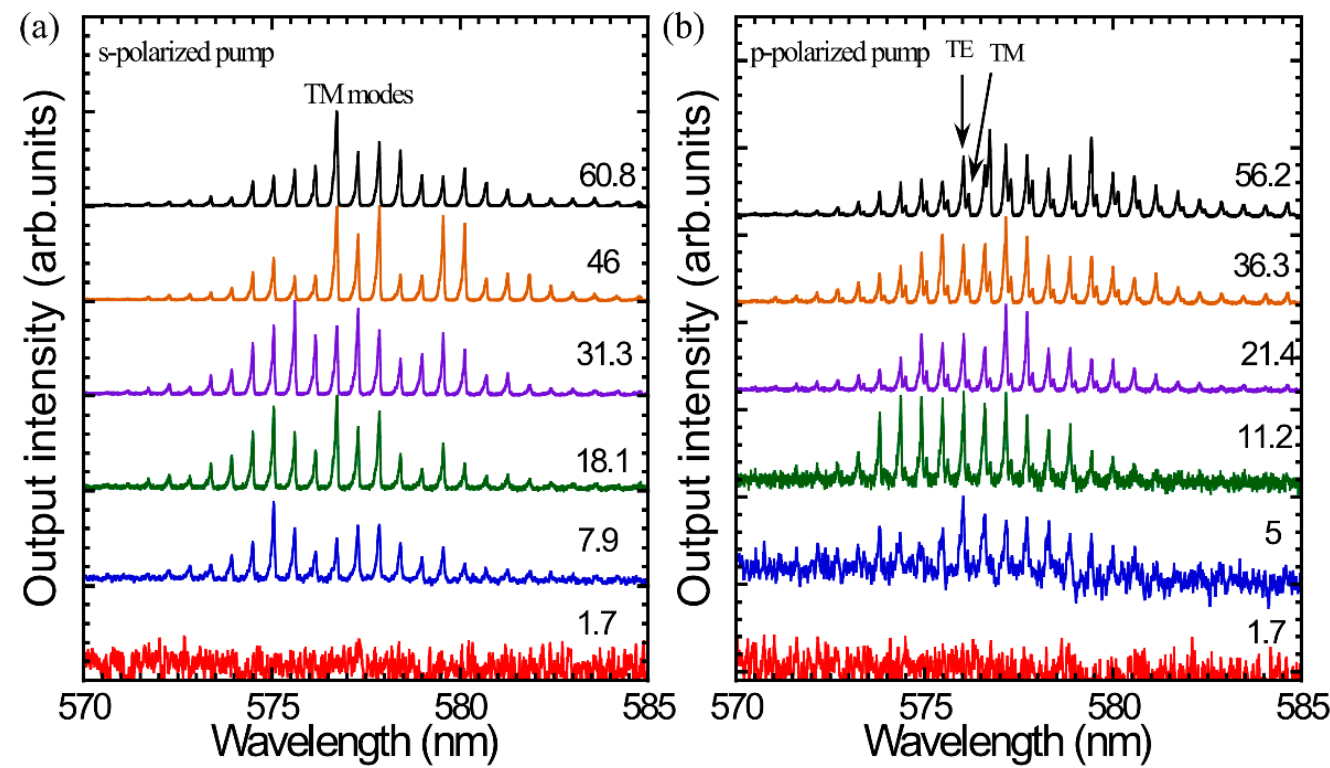

Figure 2. Multimode emission spectra of a fiber in $2.5 \mathrm{mg} / \mathrm{mL}$ Rh6G ethanol solution, at various excitation intensities, under s-polarized pump (a) and p-polarized pump (b), respectively. The polarization of the modes is marked in the spectrum.

The laser line spacing of multimode WGMs, $\Delta \lambda$, can be calculated by the following:

$$
\Delta \lambda=\frac{\lambda^{2}}{\pi n_{1} D}
$$

where $\lambda$ is the wavelength of the output laser, $n_{1}$ is the refractive index (RI) of the fiber and $D$ is the diameter of the fiber [24]. This value is very close to the $\Delta \lambda$ measured in Figure 2 $(0.57 \mathrm{~nm})$.

As shown in Figure 2b, when the sample was pumped by a $p$-polarized laser, as the pump energy exceeded $5 \mu \mathrm{J} /$ pulse, evenly spaced TE modes emerged in the spectra. Note that the $p$-polarized excitation laser shares the same polarization direction with TE modes of the emission. However, when the pump energy exceeded $21.4 \mu \mathrm{J} /$ pulse, another set of lasing lines with TM modes emerged in the spectra, at a longer wavelength, compared to that of TE modes, with its intensity much weaker than that of TE modes. The line spacing $\delta \lambda$ between the adjacent TE and TM modes is $0.14 \mathrm{~nm}$, being agreement with the estimated separation, which is calculated as follows:

$$
\delta \lambda=\frac{\lambda^{2}\left[1-\left(n_{2} / n_{1}\right)^{2}\right]^{\frac{1}{2}}}{\pi n_{1} D}
$$


where $n_{1}$ denotes the RI of the fiber (1.455) and $n_{2}$ denotes the RI of the dye solution $(1.361)[28,29]$. It is a little bit surprising that the TM modes emerged with s-polarized excitation, since it had been concluded in References [21-24] that the polarization property of lasing emission is simply dependent on the polarized state of the pump light in the sidepumping scheme. However, we also noted that the conclusion simply is from perfect ring resonator, not like in the current work, in which there is a possible leakage of evanescent wave to the wall of cuvette. This would be the key factor to understand this phenomenon.

On the other hand, when the fiber is sticking to the cuvette tightly, i.e., the optical coupling between fiber/cuvette could dominate the leakage loss of evanescent wave in the fiber, the single mode WGM emission would be observed, as shown in Figure 3.
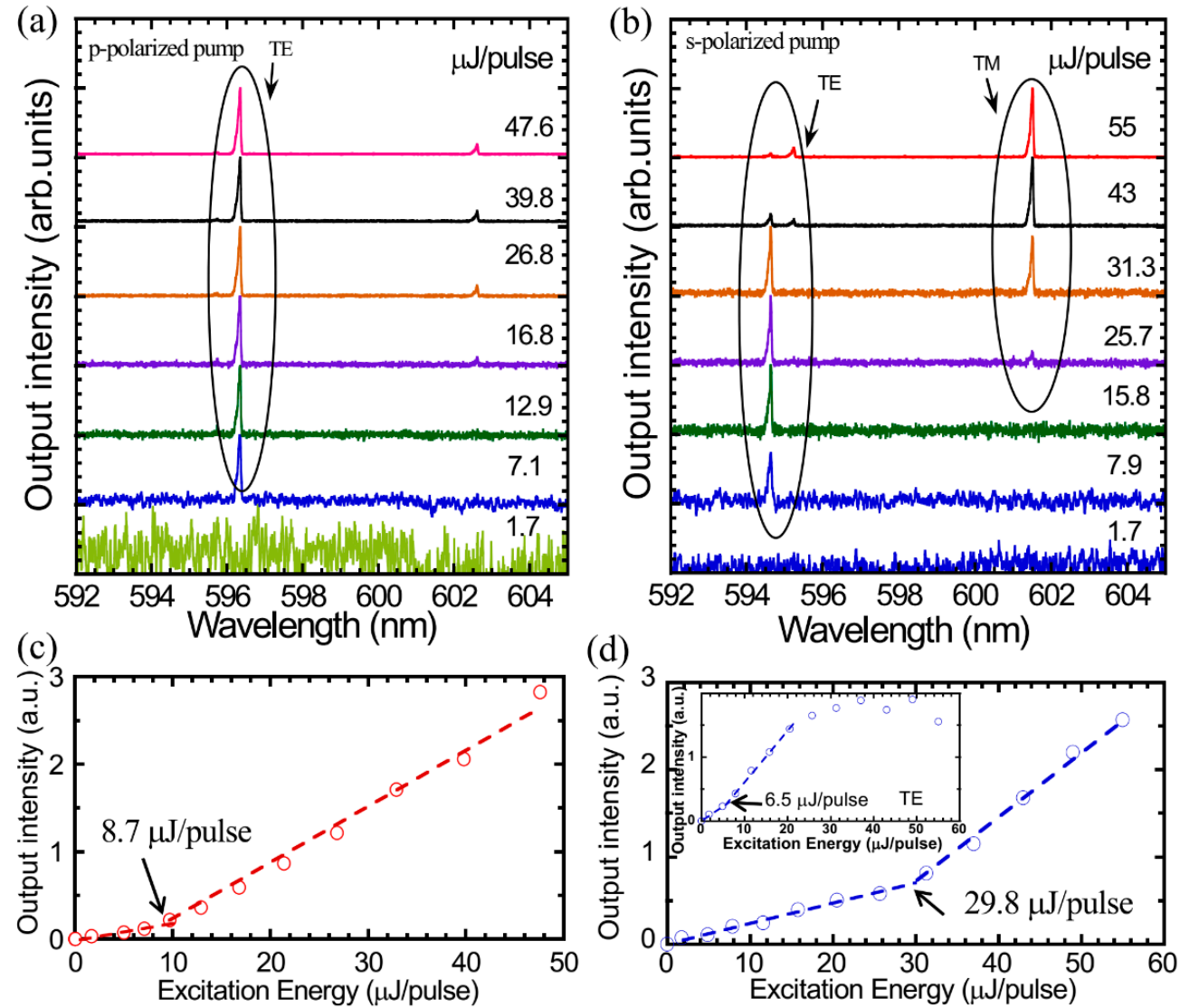

Figure 3. Single-mode emission spectra from a fiber in $2.5 \mathrm{mg} / \mathrm{mL}$ Rh6G ethanol solution under p-polarized pump (a) and s-polarized pump (b) at various pump energies; the polarization of the modes are marked in the spectrum. Integrated intensity of the emission as the function of excitation pulse energy under p-polarized pump (c) and s-polarized pump (d); (d) inset shows integrated intensity of the TE modes as the function of excitation pulse energy.

Figure 3a shows that, when the fiber is pumped by $p$-polarized light, the lasing peak with TE polarization firstly emerges at $596 \mathrm{~nm}$, at pump energy of $7.1 \mu \mathrm{J} /$ pulse, and it becomes stronger with the increasing of the pump. When the pump energy reaches $16.8 \mu \mathrm{J} /$ pulse, the second lasing line with TM polarization (vertical to the pump) emerges at $603 \mathrm{~nm}$, with much weaker intensity. When the pump energy is further increased to $47.6 \mu \mathrm{J} /$ pulse, the TE mode maintains much stronger than the TM mode in the spectrum. This is very close to the spectral features of multimode condition shown in Figure $2 b$.

Figure $3 \mathrm{~b}$ shows the emission spectrum when the fiber is pumped by the $s$-polarized laser. To our surprise, at the pump energy of $7.9 \mu \mathrm{J} /$ pulse, the first laser line emerging at $594.67 \mathrm{~nm}$ was TE polarized, which was vertical to the polarization of pump. 
When the pump energy was increased to around $29 \mu \mathrm{J} /$ pulse, the second lasing line with TM polarization emerged at $601.57 \mathrm{~nm}$, with its intensity equal to that of TE mode at $31.3 \mu \mathrm{J} /$ pulse. When the pump energy further increased to $43 \mu \mathrm{J} /$ pulse, the $601.57 \mathrm{~nm}$ lasing line with TM polarization became much stronger and then dominated the spectrum. Figure $3 c$,d shows the integrated emission intensities varying with pump energies under the $p$ - and s-pump, respectively. The lasing threshold is approximately $8.7 \mu \mathrm{J} /$ pulse for TE lasing under $p$-polarized pump, while it is $6.5 \mu \mathrm{J} /$ pulse for TE lasing and $29.8 \mu \mathrm{J} /$ pulse for TM lasing under s-polarized pump.

We can conclude from the experimental results that, in the multimode situation, the polarization of the lasing line is mainly determined by the polarization of the pump light. The lasing modes are mainly TM polarization when pumped by s-polarized light, and TE polarization when pumped by $p$-polarized light. However, in the single-mode situation, no matter what the polarization of the excitation beam is, the emission would be single TE-WGM first above the lasing threshold. As the pump energy further increases, when the sample is excited by s-polarized lasing, the TM single line emerges and becomes dominant in the spectrum, while the TE single line will keep dominant while it is excited by $p$-polarized lasing.

For the "perfect" cylinder of fiber, the TE modes and TM modes are both eigenmodes [30]; therefore, there would be no exchange between them. However, our previous work had suggested that the single-mode emission was attributed to the loss modulation due to the optical leakage from the fiber to the cuvette front wall, which selectively amplified one or a few of the WGMs. Therefore, at the contacting area, since the leakage, the TM and TE modes of the fiber would have "interaction" between them, i.e., the TM modes could transfer to TE modes, or vice versa [31,32]. The lasing line near the center of the gain spectrum could get the highest gain, which suppresses the other modes due to depletion of the inversion population. The experimental results and calculations have suggested that TE modes have lower $Q$ values than that of TM modes $[23,28]$ in a disk cavity. This means that the TE-WGMs are more likely to leak than the TM-WGMs when the fiber is attached to the cuvette wall, so this will leave smaller number of TE modes than that of TM modes in the cavity. Therefore, it is possible that a TE mode satisfies the lasing condition easier, because the lack of TE modes will make more excitons available for lasing. Thus, a single TE-WGM is more likely to be selected than TM-WGM even under s-polarized pump just above lasing threshold, because of the existence of the interaction between TE and TM modes in fiber. However, with the increase of excitation power, when a single TM-WGM is also above the lasing threshold, because of its larger $Q$ value, the energy could be easily built up in TM mode, resulting in the saturation and even lead to the reduction of the TE emission, as shown in Figure 3b.

Such an "interaction" also exists (but weak) at multimode emission condition shown in Figure 2 that could explain the appearance of TM emission excited by $p$-polarized laser, since TM modes have larger $Q$ values.

In addition, we also investigated the effect of the dye solution concentration on the single mode, under s-polarized and $p$-polarized excitation, respectively. As shown in Figure 4a, when the fiber was pumped by $p$-polarized light, at pump energy of $12.9 \mu \mathrm{J} /$ pulse, the single mode with TE polarization had a blue shift from 594 to $587.6 \mathrm{~nm}$ when increasing the solution concentration from 1 to $10 \mathrm{mg} / \mathrm{mL}$.

When the fiber was pumped by s-polarized light, at pump energy of $49 \mu \mathrm{J} / \mathrm{pulse}$, both TE and TM single modes existed in the spectra, with the TM mode being much stronger than the TE mode; the TM modes had a blue shift from 600.9 to $594.9 \mathrm{~nm}$, as the concentration of the solution was increased from 1 to $10 \mathrm{mg} / \mathrm{mL}$. This suggests that the central lasing wavelength of the single-mode lasing had a blue shift as the concentration of the solution was increased, proving that the wavelength of the single-mode lasing can be tuned. 

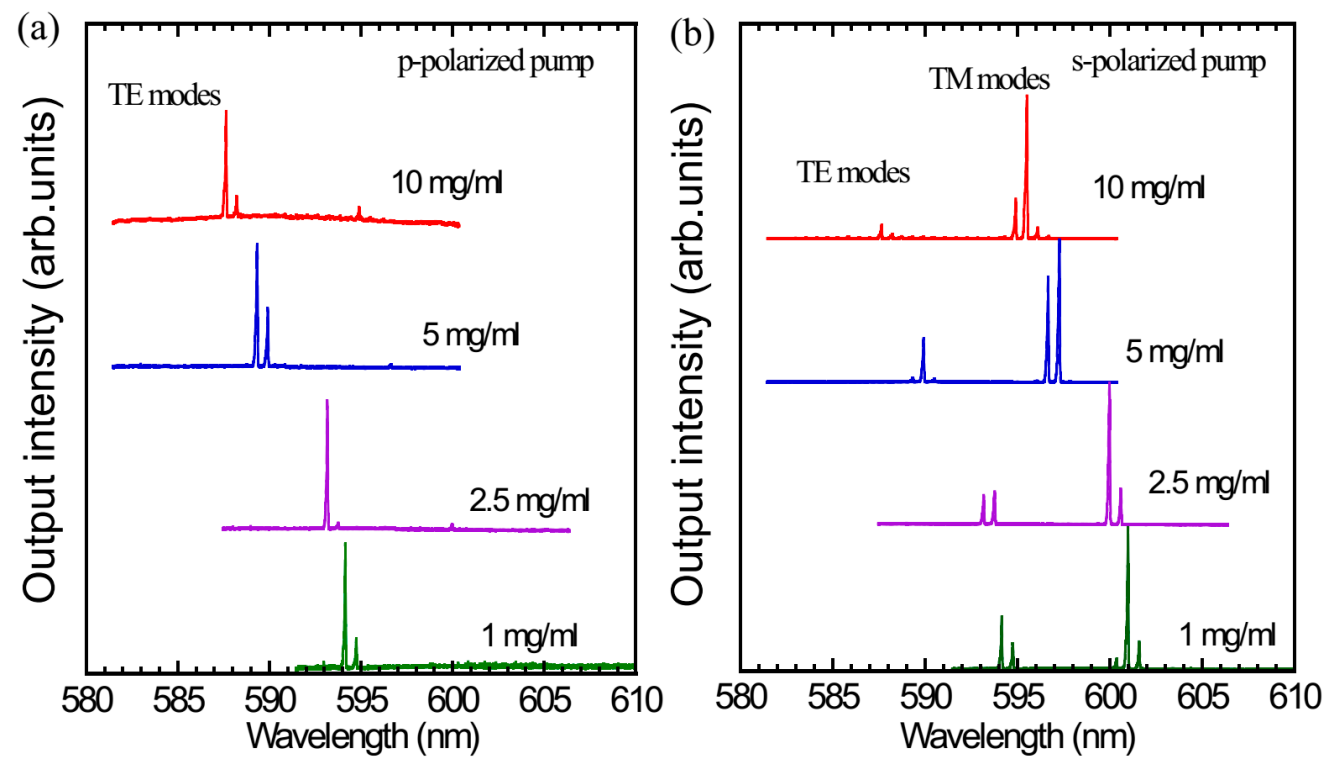

Figure 4. Single-mode WGM spectra of a fiber in Rh6G ethanol solution with different concentrations under $p$-polarized pump at the pump energy of $12.9 \mu \mathrm{J} /$ pulse (a); under s-polarized pump at the pump energy of $49 \mu \mathrm{J} /$ pulse (b). The corresponding concentration is marked at each spectrum.

Intuitively, a higher-concentration device would be expected to have a red-shift spectrum, as compared to a lower concentration one, because the increased self-absorption in higher-concentrated solution will result in the red-shift of the gain spectrum normally. However, the single-mode emission results from the losses due to the fiber attaching to the cuvette. Although understanding the reason for blue-shift of the single-mode emission is out of the scope of current work, the detailed analysis in spectra of gain and loss, as well as in competition between the losses to cuvette and to solution, would be part of the key factors to understanding this phenomenon. Nevertheless, the spectral blue-shift at higher concentration solutions would be another unique property in single-mode emission of WGMs laser.

\section{Conclusions}

In conclusion, using a fiber of $125 \mu \mathrm{m}$ in diameter and a dye solution, we observed both controllable multi- and single-WGM optofluidic lasers based on evanescent-wave-coupled gain. When the laser emission is multimode, where the leakage from fiber to cuvette is weak, i.e., the laser cavity is close to ideal disk (fiber cross section), lasing emission with almost pure TM (TE) modes is obtained when the pump laser has the electric field parallel (perpendicular) to the fiber axis. On the other hand, when the laser emission is single-mode, where the leakage from fiber to cuvette is relatively strong-i.e., the laser cavity is more like a disk with a defect, in which the energy exchange of TE and TM modes would be possiblethe single line of TE modes always emerges first above the lasing threshold, and then the TE emission continues while the pump laser's intensity increases with polarization direction perpendicular to the fiber axis. On the contrary, TE emission will dwindle relatively while the TM emission emerges and dominates the spectra when the pump laser's intensity increases with polarization parallel to the fiber axis. Furthermore, the concentration of lasing materials solution can also be used to adjust the emission wavelength. Our work proves that controlling the leakage of the evanescent wave from high-Q microcavities will be crucial not only for achieving desired optofluidic lasers with controllable multi- and single-mode emission, but also for controlling the output polarization of microfiber lasers.

Author Contributions: Conceptualization, Y.W., H.L. (Hongsheng Li) and C.S.; methodology, Y.W. and H.L. (Hongsheng Li); software, S.H.; validation, Y.W. and H.L. (Hongsheng Li); formal analysis, C.S., Y.W. and H.L. (Hongsheng Li); investigation, Y.W. and H.L. (Hongsheng Li); resources, C.S. 
and H.L. (Hongsheng Li); data curation, C.S., S.H. and Y.W.; writing-original draft preparation, Y.W. and H.L. (Hongsheng Li); writing-review and editing, C.S. and H.L. (Heng Li); visualization, Y.W., H.L. (Hongsheng Li) and S.H.; supervision, C.S. and H.L. (Heng Li); project administration, C.S.; funding acquisition, C.S. and Y.W. All authors have read and agreed to the published version of the manuscript.

Funding: This research was funded by Natural Science Foundation of Shandong Province, China, grant number ZR2019BF050 and grant number ZR2019YQ01; National Natural Science Foundation of China, grant number 11975132.

Data Availability Statement: Not applicable.

Conflicts of Interest: The authors declare no conflict of interest.

\section{References}

1. Chen, J.H.; Shen, X.; Tang, S.J.; Cao, Q.T.; Xiao, Y.F. Microcavity Nonlinear Optics with an Organically Functionalized Surface. Phys. Rev. Lett. 2019, 123, 173902. [CrossRef]

2. Zhang, X.; Cao, Q.T.; Wang, Z.; Liu, Y.X.; Qiu, C.W.; Yang, L.; Gong, Q.; Xiao, Y.F. Symmetry-breaking-induced nonlinear optics at a microcavity surface. Nat. Photon. 2019, 13, 21-24. [CrossRef]

3. Chen, W.; Jing, Z.; Bo, P.; Özdemir, S.K.; Fan, X.; Lan, Y. Parity-time-symmetric whispering-gallery mode nanoparticle sensor. Photon. Res. 2018, 6, A23-A30. [CrossRef]

4. Chen, Y.J.; Schoeler, U.; Huang, C.H.B.; Vollmer, F. Combining whispering-gallery mode optical biosensors with microfluidics for real-time detection of protein secretion from living cells in complex media. Small 2018, 14, 1703705. [CrossRef]

5. Zhi, Y.; Yu, X.C.; Gong, Q.; Yang, L.; Xiao, Y.F. Single nanoparticle detection using optical microcavities. Adv. Mat. 2017, 29, 1604920. [CrossRef] [PubMed]

6. Liu, W.; Li, M.; Guzzon, R.S.; Norberg, E.J.; Parker, J.S.; Lu, M.; Coldren, L.A.; Yao, J. An integrated parity-time symmetric wavelength-tunable single-mode microring laser. Nat. Comm. 2017, 8, 15389. [CrossRef] [PubMed]

7. Xu, C.; Qin, F.; Zhu, Q.; Lu, J.; Wang, Y.; Li, J.; Lin, Y.; Cui, Q.; Shi, Z.; Manohari, A.G. Plasmon-enhanced ZnO whispering-gallery mode lasing. Nano Res. 2018, 11, 3050-3064. [CrossRef]

8. Jiang, X.F.; Zou, C.L.; Wang, L.; Gong, Q.; Xiao, Y.F. Whispering-gallery microcavities with unidirectional laser emission. Laser Photon. Rev. 2016, 10, 40-61. [CrossRef]

9. Yang, Y.D.; Tang, M.; Wang, F.L.; Xiao, Z.X.; Xiao, J.L. Whispering-gallery mode hexagonal micro-/nanocavity lasers. Photon. Res. 2019, 7, 594-607. [CrossRef]

10. Longhi, S.; Feng, L. Unidirectional lasing in semiconductor microring lasers at an exceptional point. Photon. Res. 2017, 5, B1-B6. [CrossRef]

11. Lubatsch, A.; Frank, R. Quantum Many-Body Theory for Exciton-Polaritons in Semiconductor Mie Resonators in the NonEquilibrium. Appl. Sci. 2020, 10, 1836. [CrossRef]

12. Deng, H.; Weihs, G.; Santori, C.; Bloch, J.; Yamamoto, Y. Condensation of semiconductor microcavity exciton polaritons. Science 2002, 298, 199-202. [CrossRef] [PubMed]

13. Kasprzak, J.; Richard, M.; Kundermann, S.; Baas, A.; Jeambrun, P.; Keeling, J.M.; Marchetti, F.M.; Szymanska, M.H.; Andre, R.; Staehli, J.L.; et al. Bose-Einstein condensation of exciton polaritons. Nature 2006, 443, 409-414. [CrossRef] [PubMed]

14. Ram, R.J.; Pau, S.; Yamamoto, Y. Nonequilibrium condensates and lasers without inversion: Exciton-polariton lasers. Phys. Rev. A 1996, 53, 4250-4253.

15. Feng, L.; Wong, Z.J.; Ma, R.-M.; Wang, Y.; Zhang, X. Single-mode laser by parity-time symmetry breaking. Science 2014, 346, 972-975. [CrossRef]

16. Hodaei, H.; Miri, M.A.; Heinrich, M.; Christodoulides, D.N.; Khajavikhan, M. Parity-time-symmetric microring lasers. Science 2014, 346, 975-978. [CrossRef]

17. Gu, F.; Xie, F.; Lin, X.; Hu, S.L.; Fang, W. Single whisperinggallery-mode lasing in polymer bottle microresonators via spatial pump engineering. Light Sci. Appl. 2017, 6, e17061. [CrossRef]

18. Wang, Y.C.; Hu, S.; Yang, X.; Wang, R.Z.; Li, H.; Sheng, C.X. Evanescent-wave pumped single-mode microcavity laser from fiber of $125 \mu \mathrm{m}$ diameter. Photon. Res. 2018, 6, 332-338. [CrossRef]

19. Lubatsch, A.; Frank, R. Behavior of Floquet Topological Quantum States in Optically Driven Semiconductors. Symmetry 2019, 11, 1246. [CrossRef]

20. Lubatsch, A.; Frank, R. Evolution of Floquet topological quantum states in driven semiconductors. Eur. Phys. J. B 2019, 92, 215. [CrossRef]

21. Moon, H.J.; Chough, Y.T.; An, K. Cylindrical microcavity laser based on the evanescent-wave-coupled gain. Phys. Rev. Lett. 2000, 85, 3161-3164. [CrossRef] [PubMed]

22. Zhang, Y.X.; Meng, W.D.; Yang, H.Y.; Chu, Y.F.; Pu, X.Y. Demonstration of polarization mode selection and coupling efficiency of optofluidic ring resonator lasers. Opt. Lett. 2015, 40, 5101-5104. [CrossRef] [PubMed] 
23. Zhang, Y.X.; Pu, X.Y.; Feng, L.; Han, D.Y.; Ren, Y.T. Polarization characteristics of Whispering-Gallery-Mode fiber lasers based on evanescent-wave-coupled gain. Opti. Express 2013, 21, 12617-12628. [CrossRef] [PubMed]

24. Ta, V.D.; Chen, R.; Sun, H. Controllable Polarization of Lasing Emission from a Polymer Microfiber Laser. Sci. Rep. 2019, 9, 17017. [CrossRef]

25. Zhang, Y.X.; Pu, X.Y.; Zhu, K.; Feng, L. Threshold property of whispering-gallery-mode fiber lasers pumped by evanescent waves. J. Opt. Soc. Am. B 2011, 28, 2048-2056. [CrossRef]

26. Shevchenko, A.; Lindfors, K.; Buchter, S.C.; Kaivola, M. Evanescent-wave pumped cylindrical microcavity laser with intense output radiation. Opt. Commun. 2005, 245, 349-353. [CrossRef]

27. Polson, R.C.; Levina, G.; Vardeny, Z.V. Spectral analysis of polymer microring lasers. Appl. Phys. Lett. 2000, 76, 3858-3860. [CrossRef]

28. Zhang, Y.X.; Li, D.Y.; Zhang, H.; Sun, Y.Z.; Chu, Y.F.; Pu, X.Y. Influence of Refractive Index on Lasing Coupling Efficiency and Emission Spectrum. IEEE Photonics Technol. Lett. 2019, 31, 1277-1280. [CrossRef]

29. Lam, C.C.; Leung, P.T.; Young, K. Explicit asymptotic formulas for the positions, widths, and strengths of resonances in mie scattering. J. Opt. Soc. Am. B 1992, 9, 1585-1592. [CrossRef]

30. Polson, R.C. Laser action in microcavities of pi-conjugated polymers. Ph.D. Thesis, The University of Utah, Salt Lake City, UT, USA, 2002.

31. Rosenberger, A.T.; Dale, E.B.; Bui, K.V.; Gonzales, E.K.; Rajagopal, S.R. Cross-polarization coupling of whispering-gallery modes due to the spin-orbit interaction of light. Opt. Lett. 2019, 44, 4163-4166. [CrossRef]

32. Bui, K.; Rosenberger, A.T. Coupled-mode-induced transparency and attenuation resulting from cross-polarization coupling. Phys. Rev. A 2020, 101, 033836. [CrossRef] 